\title{
Promotion of Color-Changing Luminescent
}

\author{
Hydrogels from Thermo to Electrical
}

\section{Responsiveness towards Biomimetic Skin}

\section{Applications}

Shuxin Wei, ${ }^{\dagger, \ddagger}$ Huiyu Qiu, ${ }^{\dagger}$ Huihui Shi, ${ }^{\dagger, \ddagger}$ Wei Lu ${ }^{*+, \neq}$ Hao Liu, ${ }^{\dagger, \ddagger}$ Huizhen Yan $^{\dagger}{ }^{\dagger}$ Dachuan

Zhang, ${ }^{\dagger}$ Jiawei Zhang, ${ }^{\dagger, \neq}$ Patrick Theato, ${ }^{\S, \|}$ Yen Wei, "and Tao Chen ${ }^{*}, \neq$

${ }^{\dagger}$ Key Laboratory of Marine Materials and Related Technologies, Zhejiang Key Laboratory of Marine Materials and Protective Technologies, Ningbo Institute of Materials Technology and Engineering, Chinese Academy of Sciences, Ningbo 315201, China.

¥School of Chemical Sciences, University of Chinese Academy of Sciences, 19A Yuquan Road, Beijing 100049, China.

§Soft Matter Synthesis Laboratory, Institute for Biological Interfaces III, Karlsruhe Institute of

Technology (KIT), Hermann-von-Helmholtz-Platz 1, D-76344 Eggenstein-Leopoldshafen, Germany.

\|Institute for Chemical Technology and Polymer Chemistry, Karlsruhe Institute of Technology (KIT), Engesser Str. 18, D-76131 Karlsruhe, Germany. 
^The Key Laboratory of Bioorganic Phosphorus Chemistry and Chemical Biology, Department of Chemistry, Tsinghua University, Beijing 100084, China.

*Corresponding author, Email: luwei@nimte.ac.cn; tao.chen@nimte.ac.cn

This PDF file includes:

Figures S1 to S21

Other Supplementary Materials for this manuscript include the following:

Movies S1 to S4 


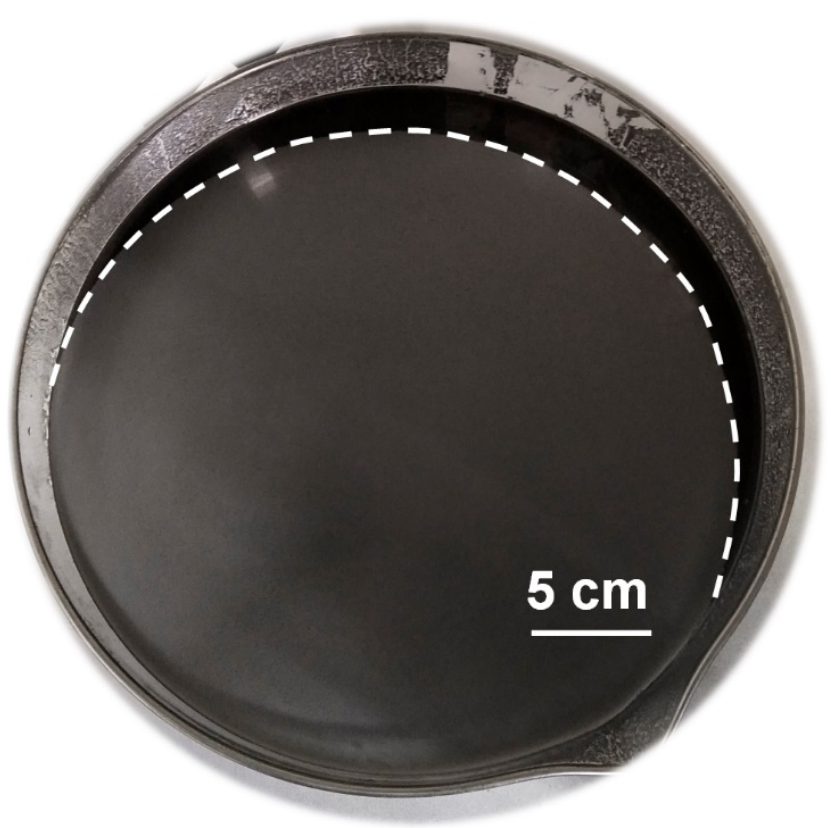

Figure S1. Digital photo of large-area closely packed SGA thin film on the surface of the water.

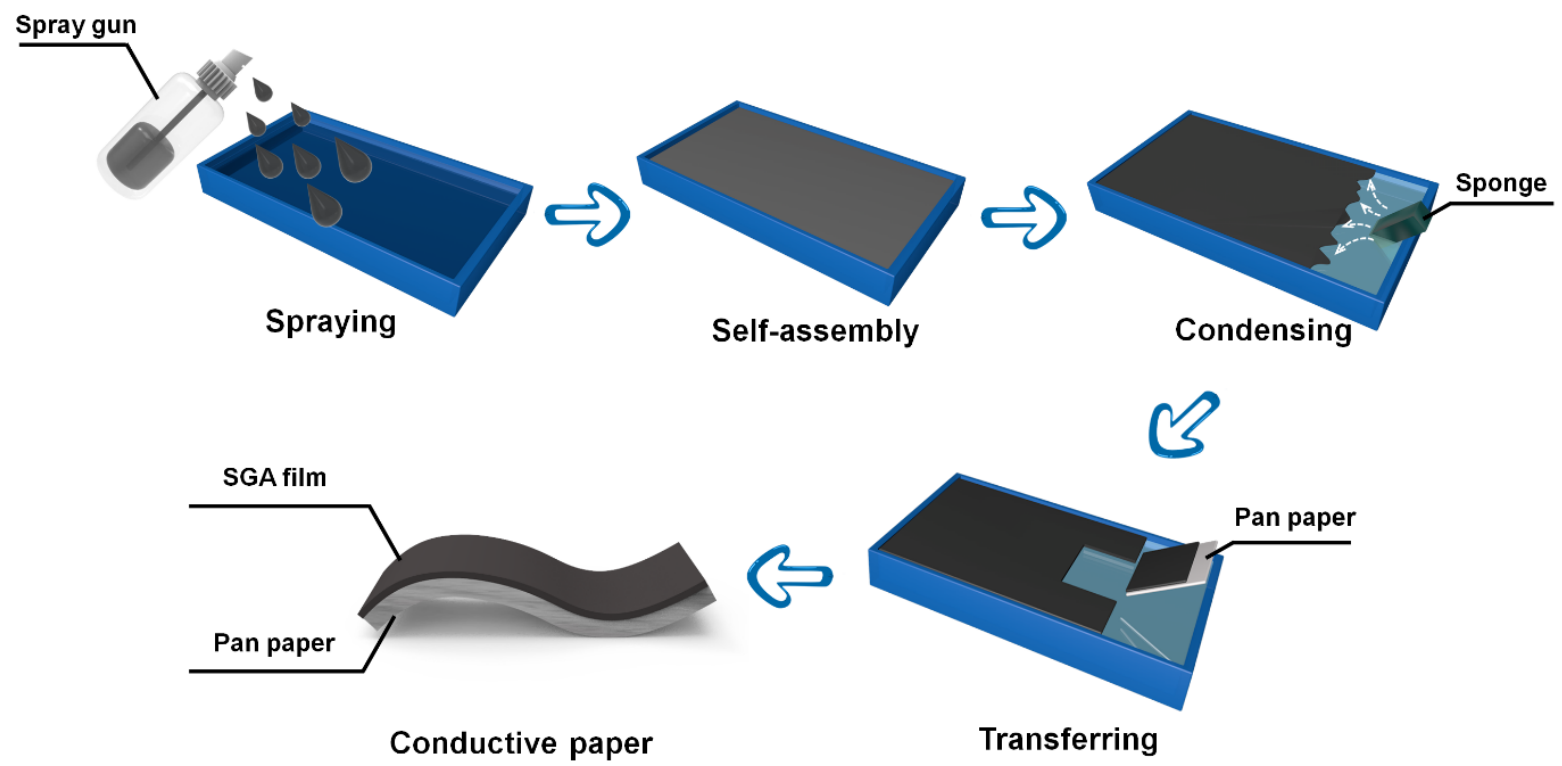

Figure S2. Sketch of the fabrication process of the conductive paper. The graphene flakes ethanol dispersions were sprayed on the surface of water to form the self-assembled graphene film. A piece of sponge is gradually inserted into the water from aside to change the surface tension, resulting in the formation of closely packed SGA film. The flexible conductive paper was then prepared by transferring such condensed SGA film onto pan paper. 


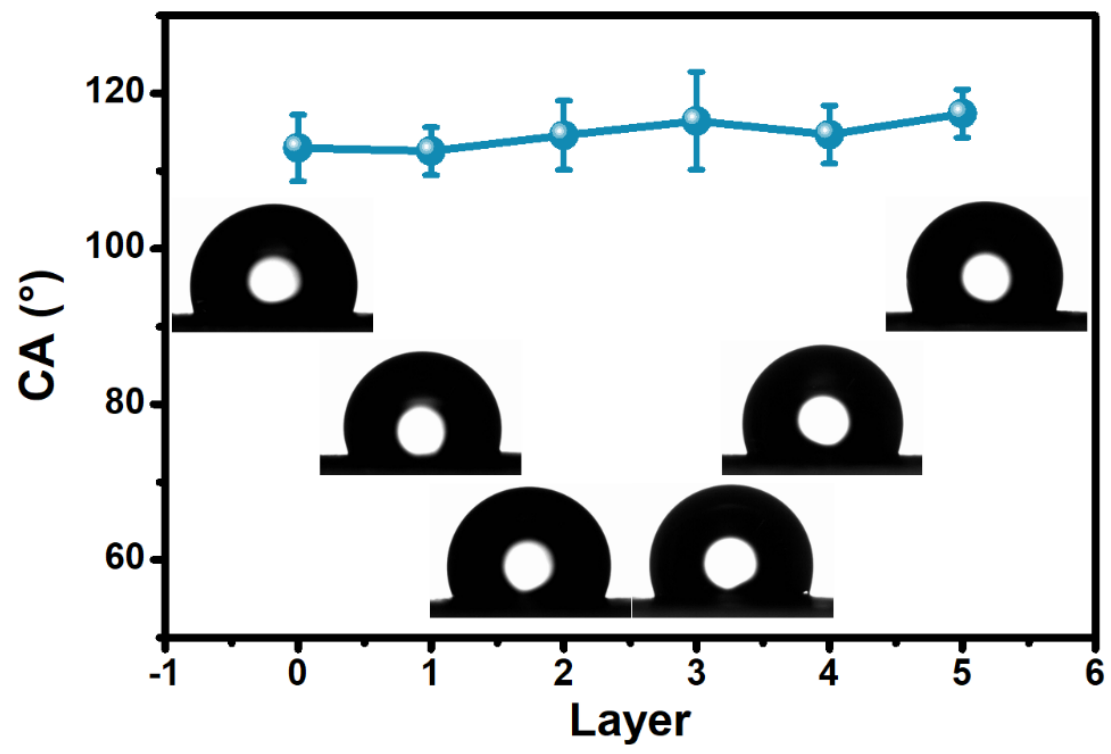

Figure S3. Water contact angles of pan paper and the conductive papers with different SGA layers (one to five layers).
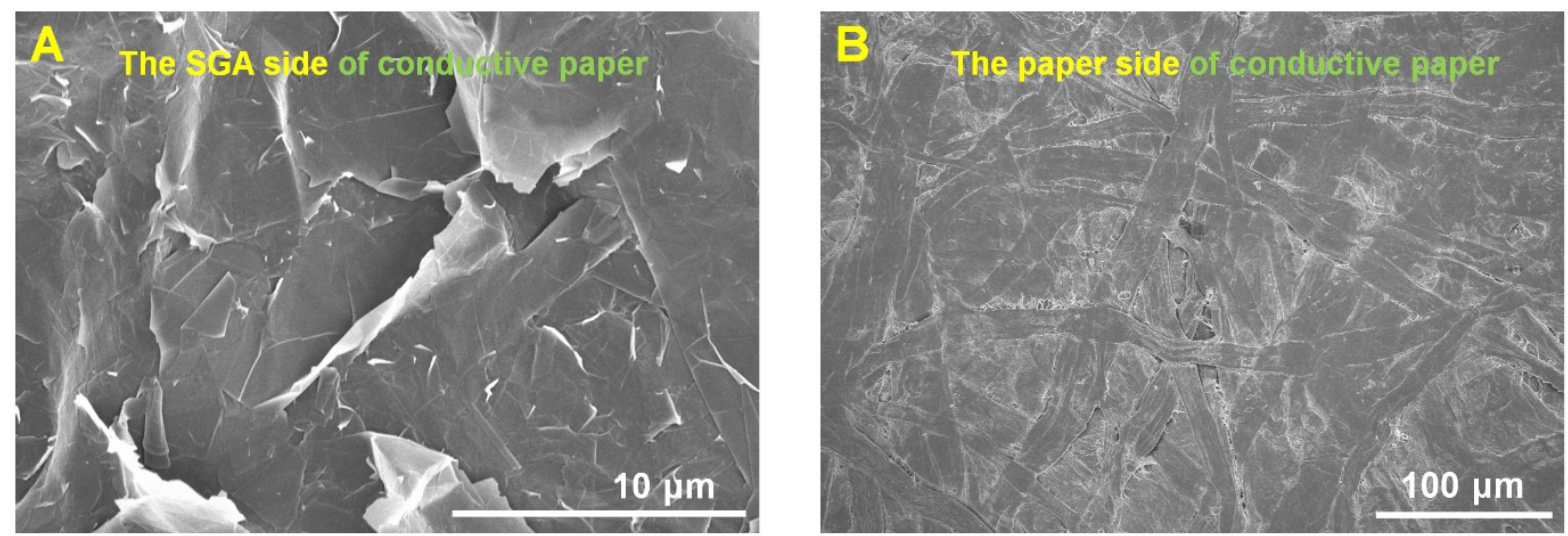

Figure S4. The microstructure and morphology of the conductive paper. SEM images of (A) the SGA film side and (B) the paper side of the conductive paper with four layers of SGA film. 

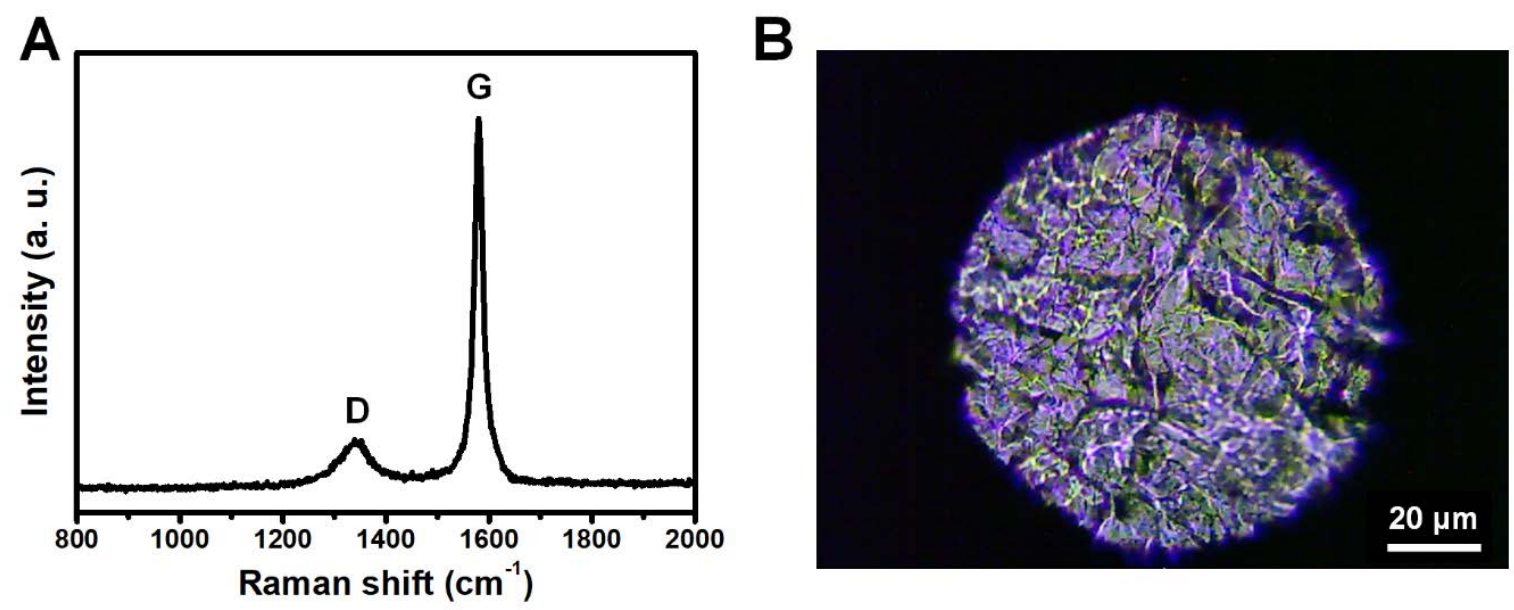

Figure S5. Raman analysis of SGA film. (A) Raman spectrum of a SGA film coated on the pan paper and (B) the corresponding photo captured by confocal microscopy.

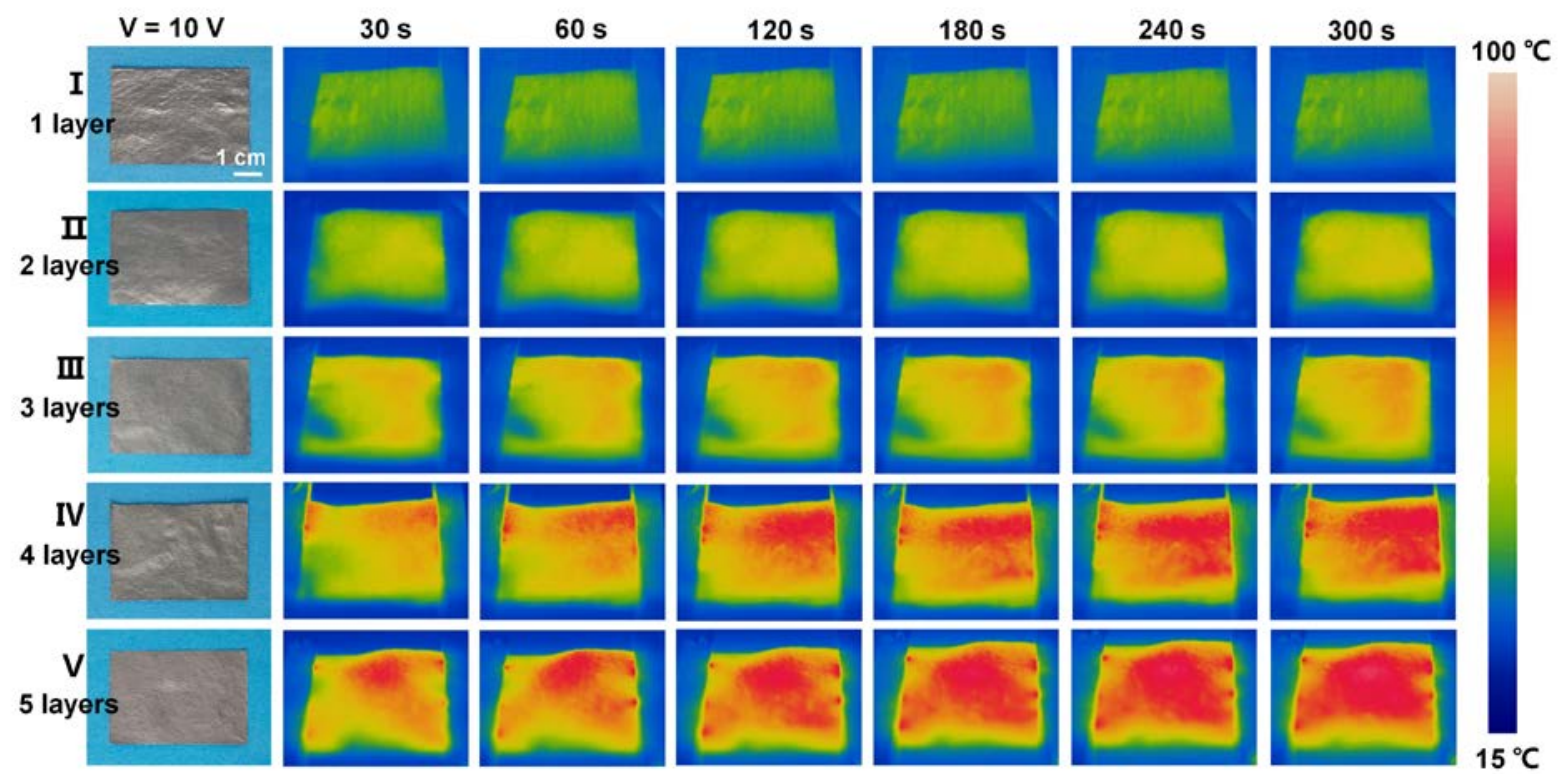

Figure S6. IR images showing the temperature increase process of the conductive papers with different SGA film layers under $10 \mathrm{~V}$. 


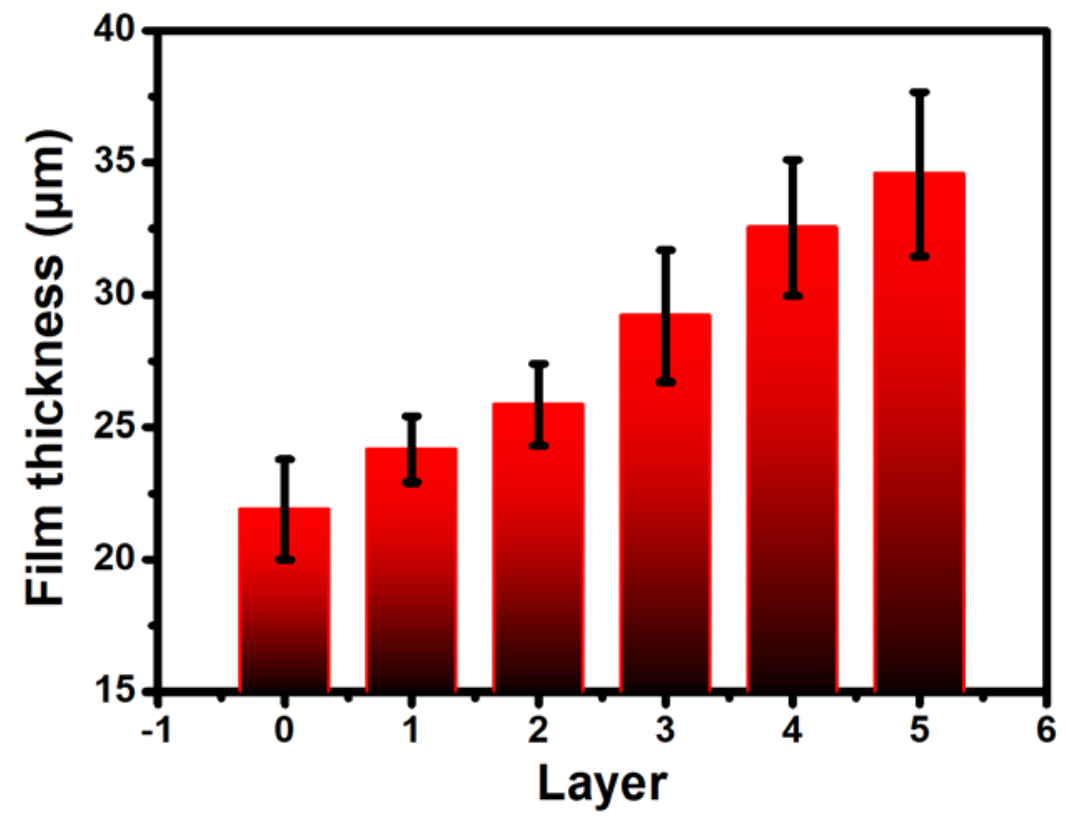

Figure S7. Film thickness of the conductive papers with different SGA film layers (zero to five layers).

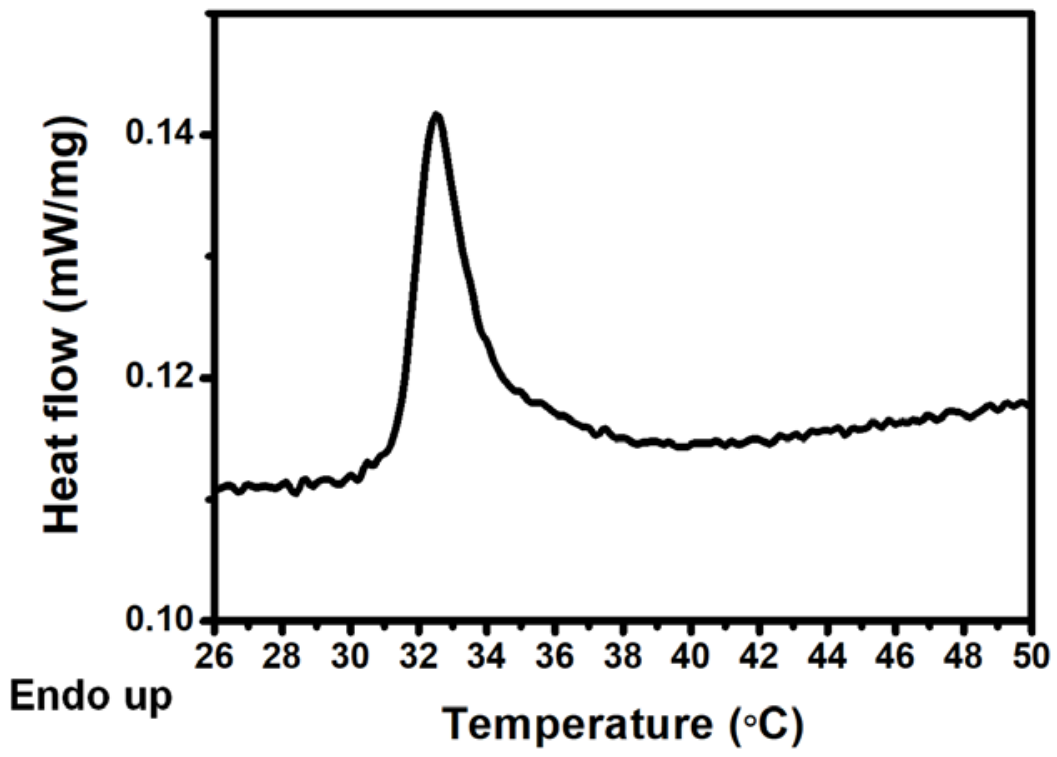

Figure S8. DSC curve of the Eu-PNIPAM hydrogel at the scanning rate of $1{ }^{\circ} \mathrm{C} \mathrm{min}-1$. 


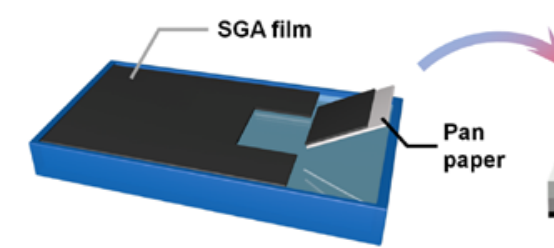

(i) Transferring graphene film to pan paper

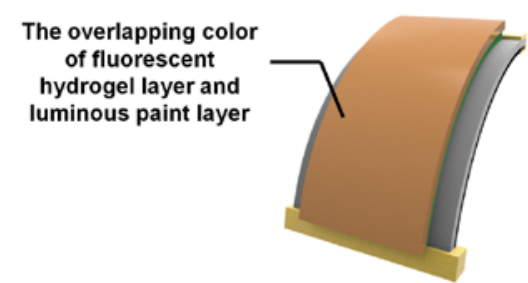

(v) Color overlay

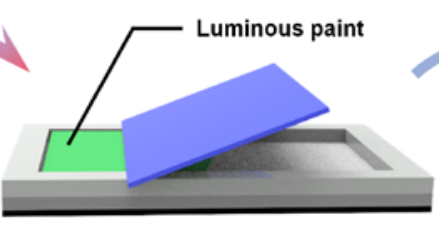

(ii) Coating luminous paint

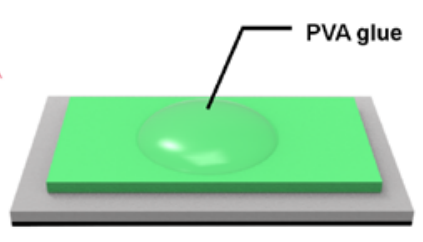

(iii) Coating PVA glue

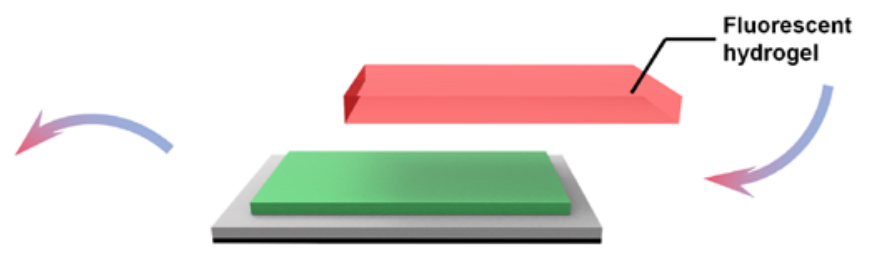

(iv) Combining fluorescent hydrogel

Figure S9. Illustration showing the fabrication process of the electrically controlled fluorescent color changeable hydrogel-graphene system. In the first step, the conductive paper was prepared by transferring the SGA film floating on air/water interface onto the pan paper. Subsequently, the luminous paint was uniformly scraped on the paper side of the conductive paper by a plastic blade. After drying the substrate, PVA glue was used to connect the as-prepared fluorescent hydrogel layer and luminous paint layer. After several freezing-thawing cycles, the electrically controlled fluorescent color changeable hydrogel-graphene system with asymmetric configuration was obtained.
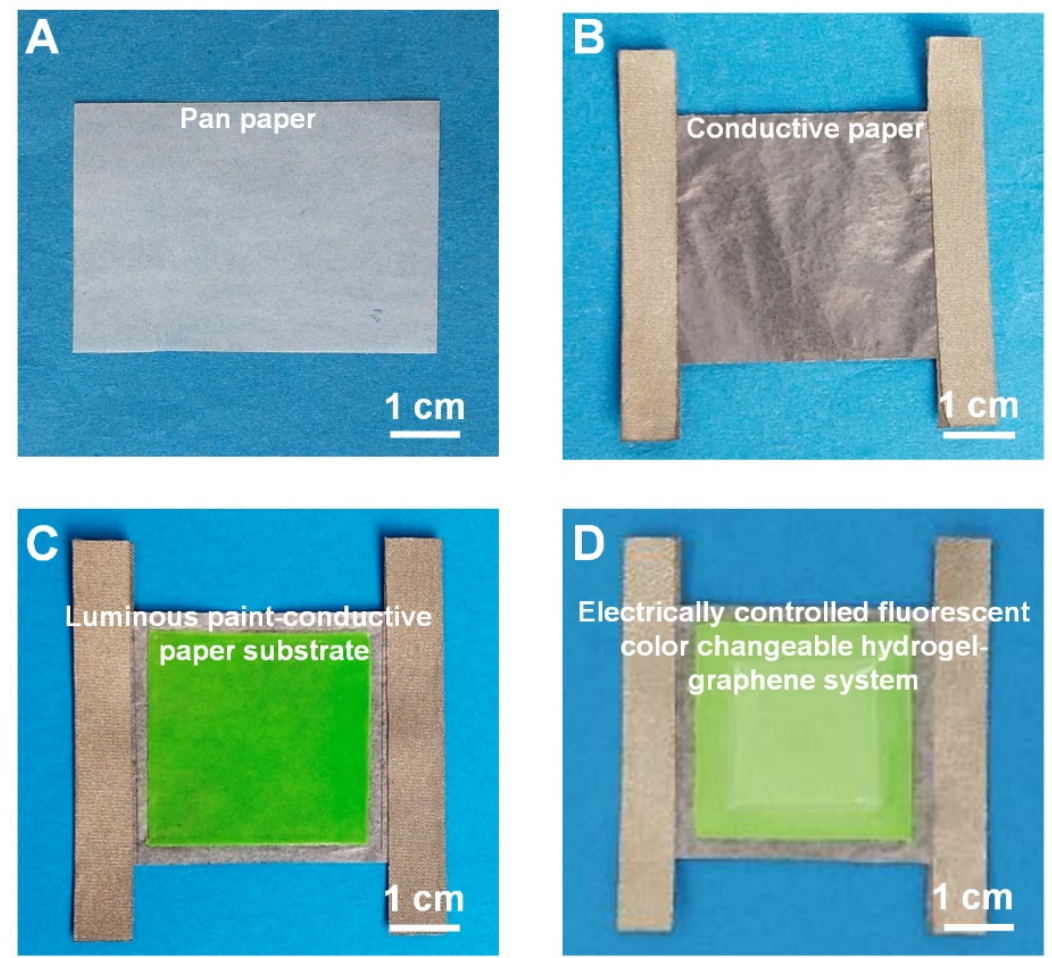

Figure S10. Photographs of pan paper, conductive paper, luminous paint/conductive paper substrate, and electrically controlled fluorescent color changeable hydrogel-graphene system. Digital photo of (A) pan paper with a dimension of $51 \times 35 \mathrm{~mm}$, (B) conductive paper with a dimension of $35 \times 35 \mathrm{~mm}$ (removing the area of electrodes), (C) luminous paint (green color)conductive paper substrate, and (D) electrically controlled fluorescent color changeable hydrogel-graphene system. 

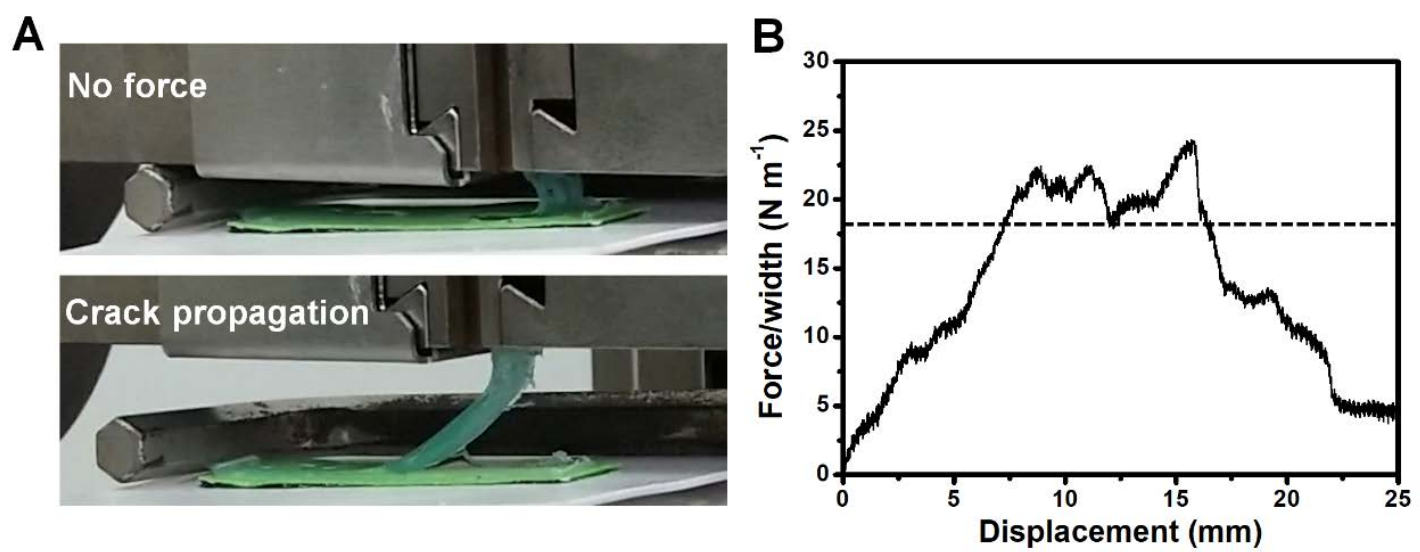

Figure S11. (A) Photos showing the peeling process of the hydrogel layer and luminous paint layer. (B) Curves of the peeling force per width of hydrogel sheet versus displacement for interface bonding. To prevent the elongation of fluorescent hydrogel along the peeling direction, its surface was covered with a thin and stiff plastic film backing. In addition, the whole device was fixed on a rigid plate to avoid the slippage of the substrate layer.

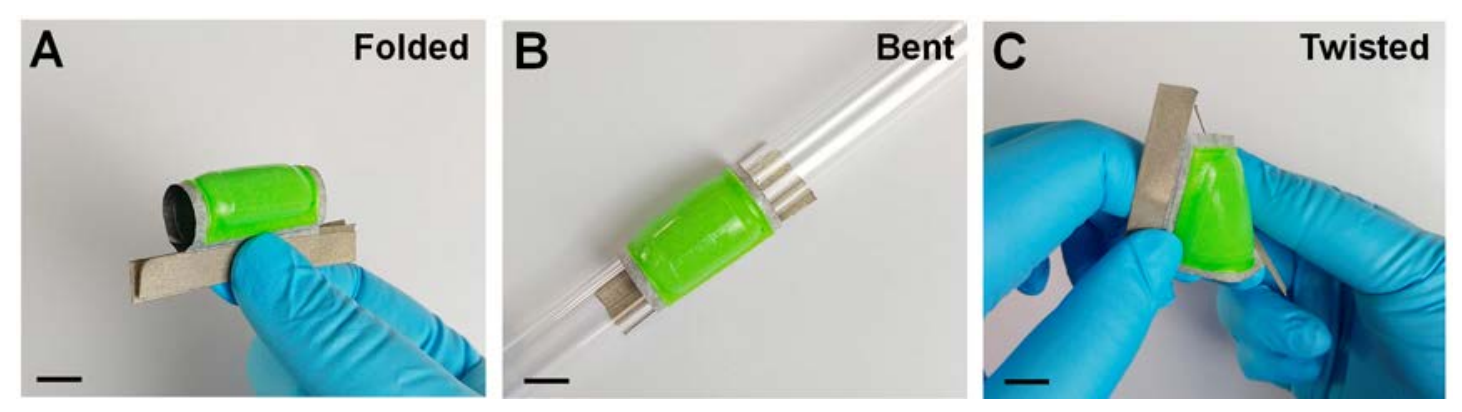

Figure S12. Photographs of the electrically controlled fluorescent color changeable hydrogelgraphene device under (A) folded, (B) bent and (C) twisted conditions. Scale bars in digital photos are $1 \mathrm{~cm}$. 

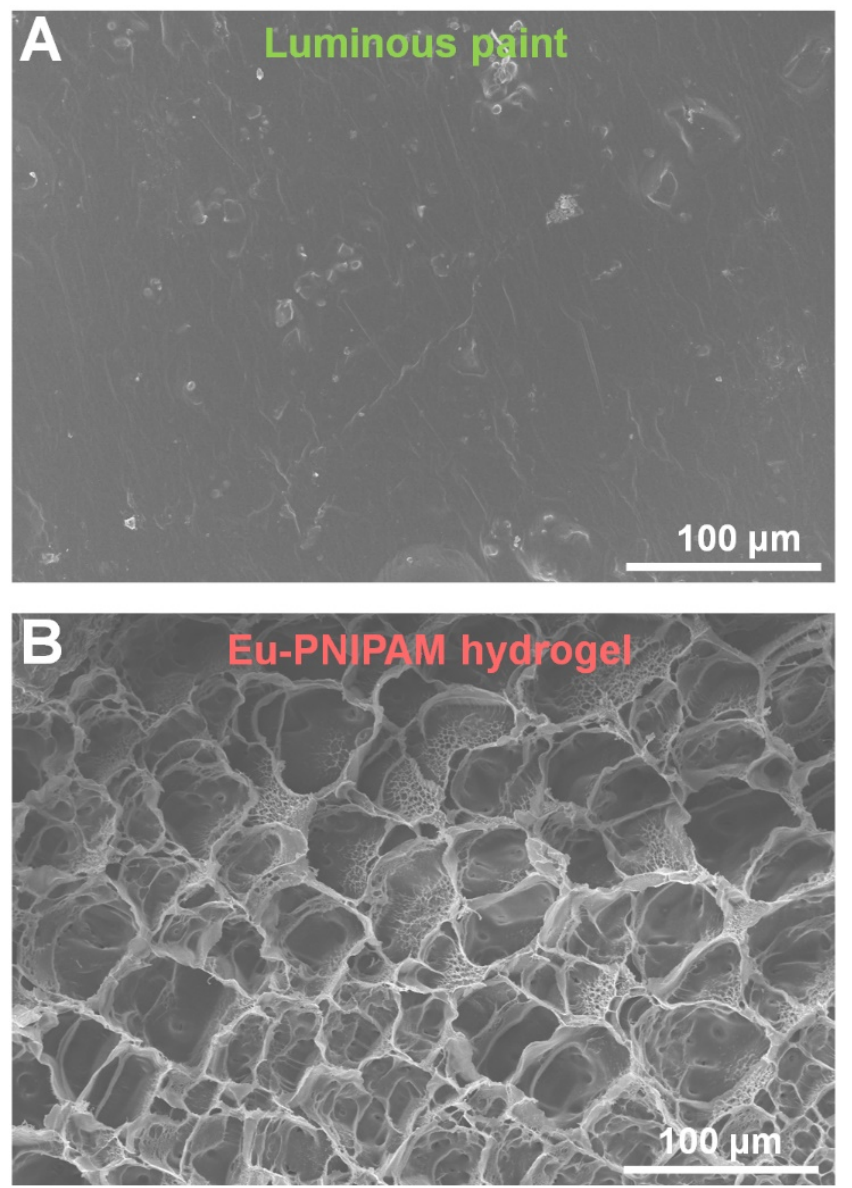

Figure S13. The microstructure of luminous paint layer and fluorescent hydrogel layer. SEM image of (A) luminous paint and (B) Eu-PNIPAM hydrogel of the electrically controlled fluorescent color changeable hydrogel-graphene system.

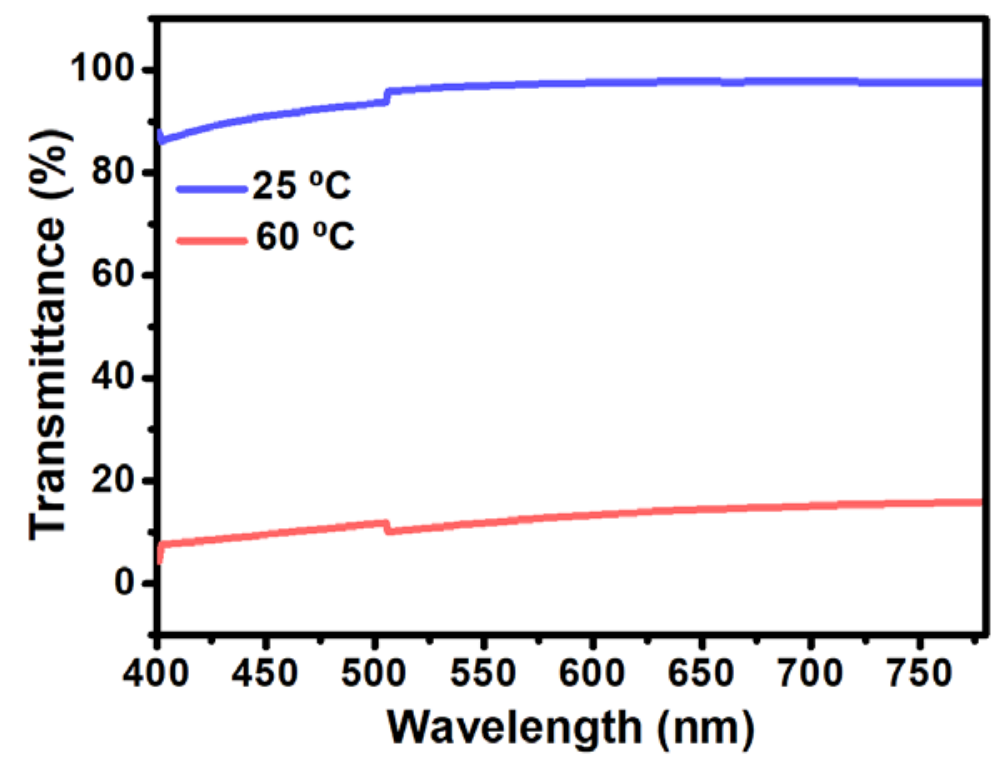

Figure S14. The transmittance spectra of the Eu-PNIPAM hydrogel at RT and $60^{\circ} \mathrm{C}$. 


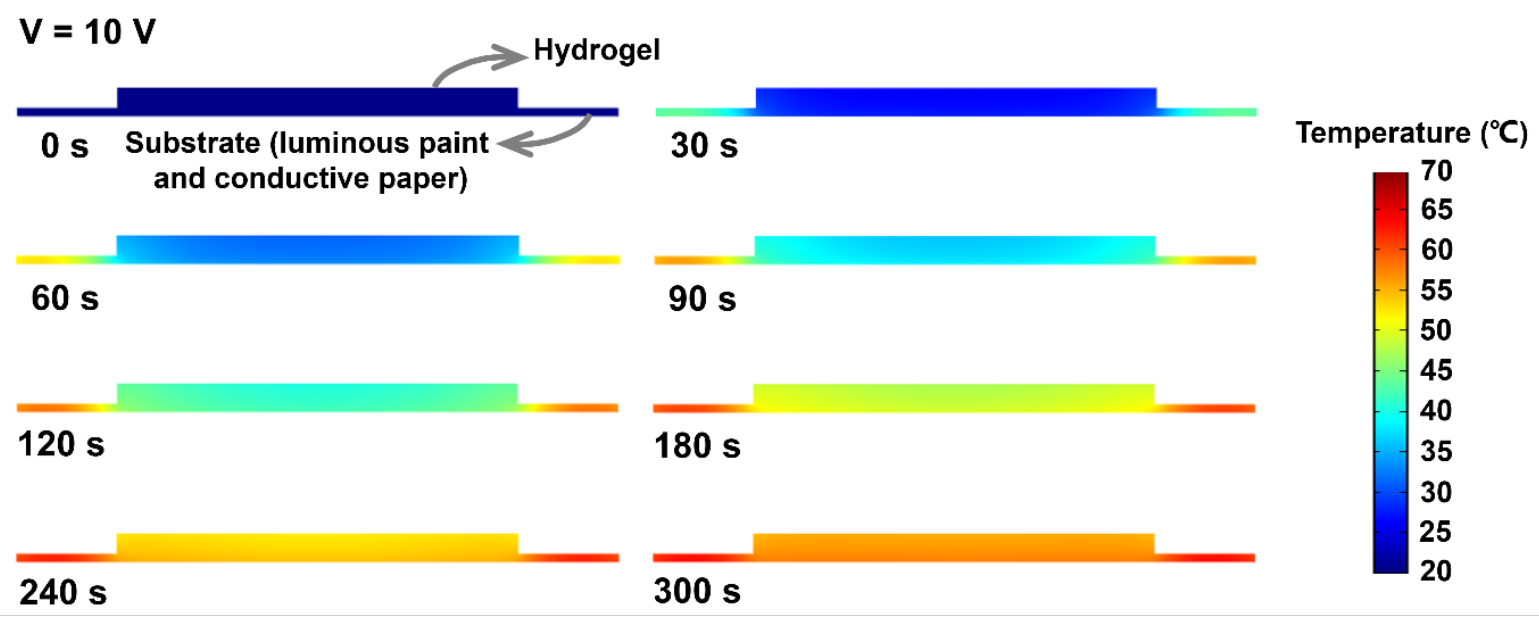

Figure S15. Finite element simulation of cross-section temperature distribution of hydrogelgraphene system with different power-on time under the voltage of $10 \mathrm{~V}$.
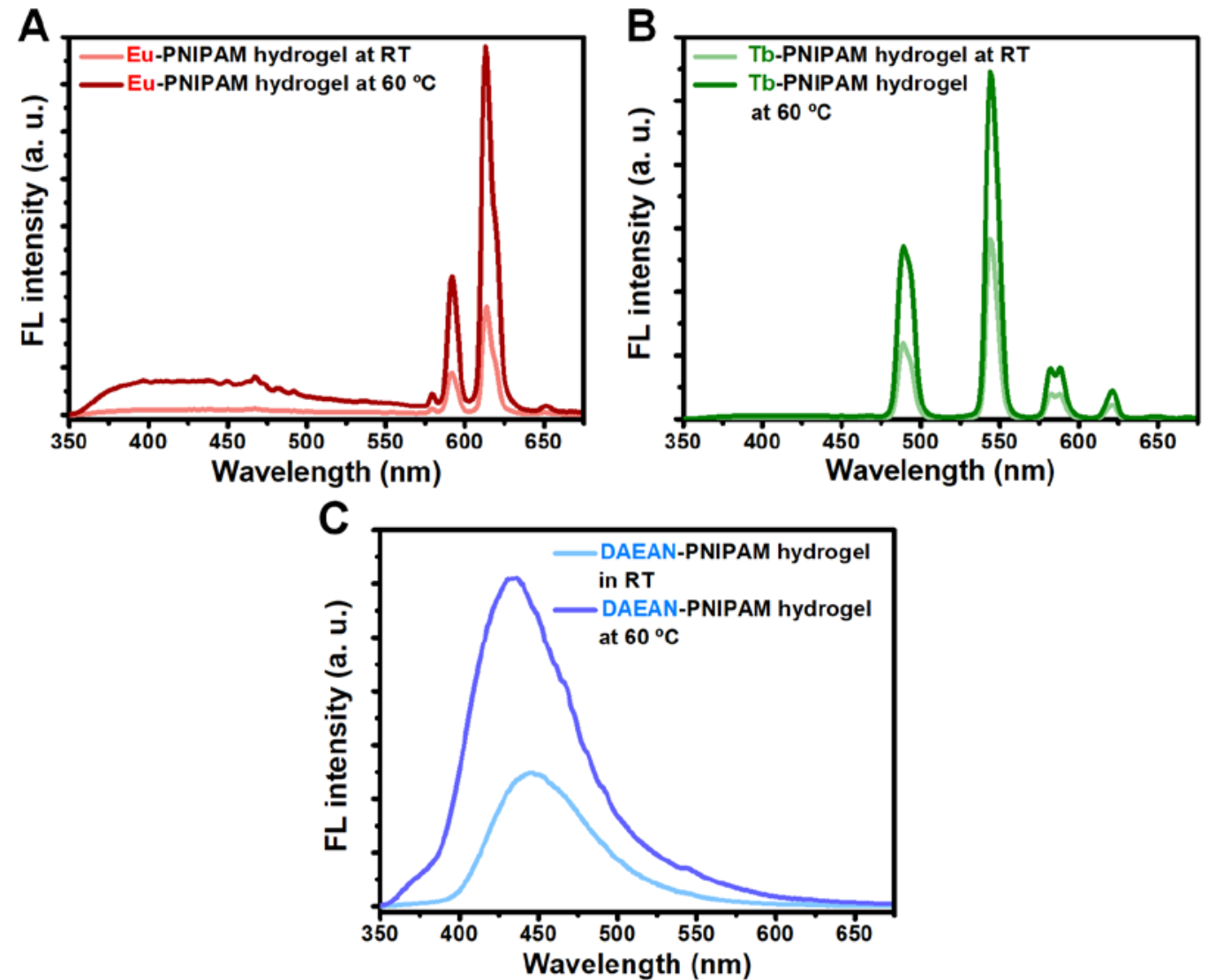

Figure S16. Fluorescence spectra $\left(\lambda_{\mathrm{ex}}=254 \mathrm{~nm}\right)$ of three different-colored fluorescent hydrogels recorded at RT and $60{ }^{\circ} \mathrm{C}$. For Eu-PNIPAM and Tb-PNIPAM hydrogels, the phase transition above VPTT led to the shrinkage of polymer network and the reduced hydration effect of the $\mathrm{Eu}^{3+}$ - or $\mathrm{Tb}^{3+}$-complexes, resulting in enhanced fluorescence intensity. For DAEANPNIPAM hydrogels, the increased fluorescence intensity at high temperature was mainly ascribed to the enhanced aggregation-induced emission (AIE) effect of the AIEgens caused by the shrinkage of polymer network. 


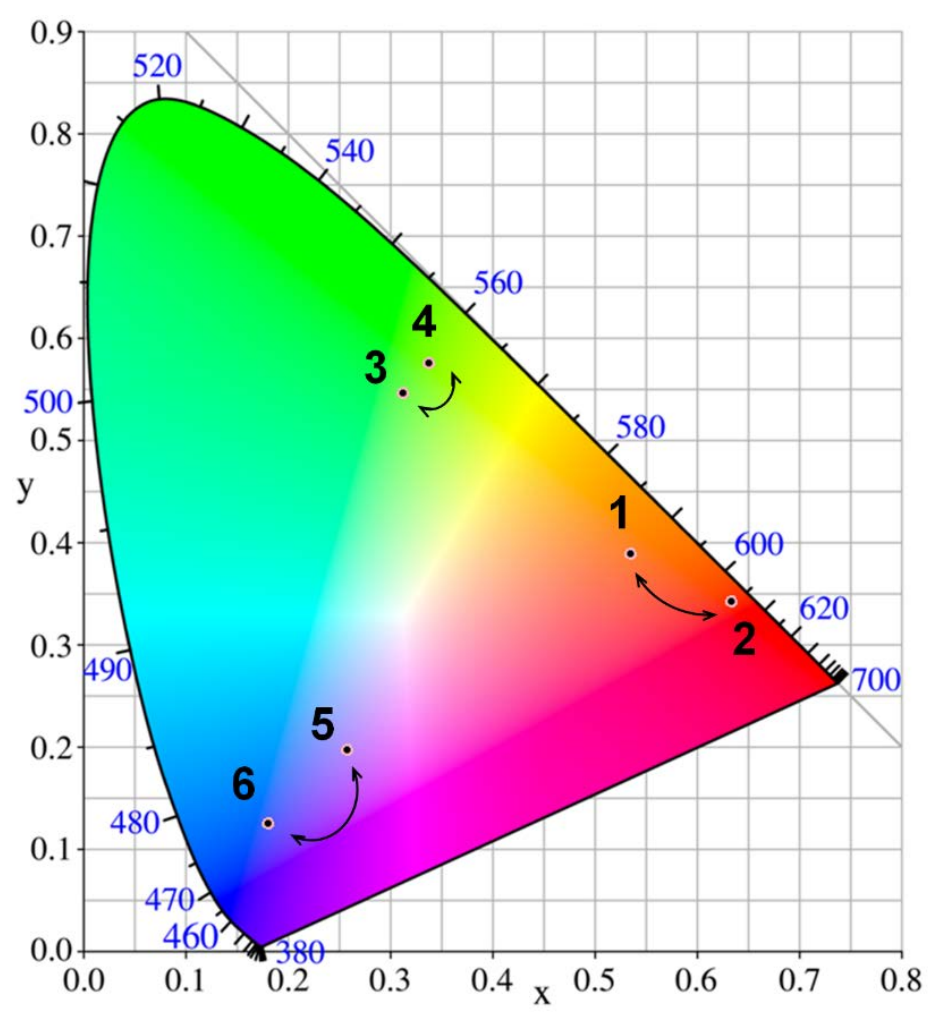

Figure S17. CIE (1931) coordinate diagrams showing the color-changing process of three electrically controlled fluorescent color changeable hydrogel-graphene system shown in Figure 4B-D. 

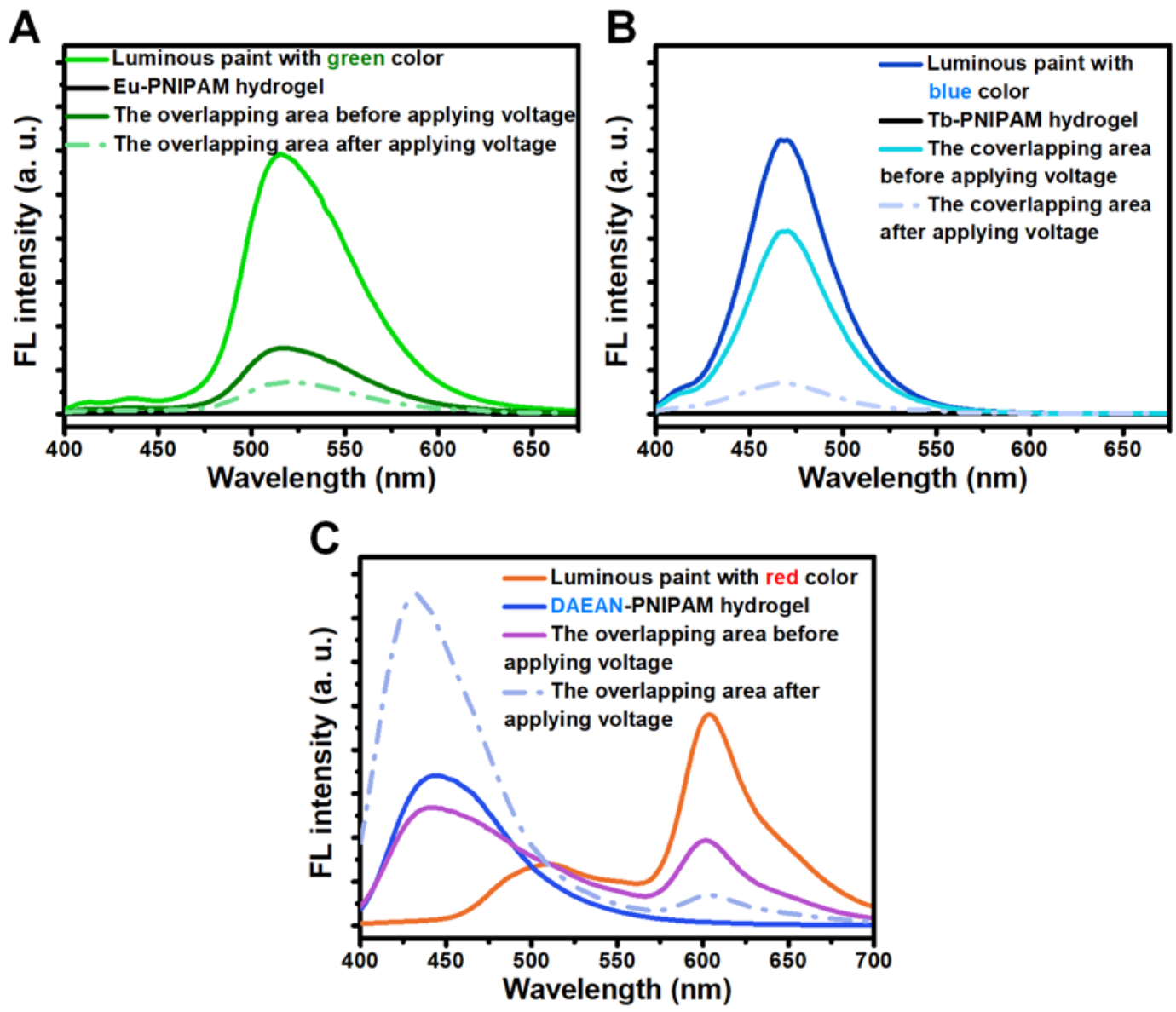

Figure S18. Fluorescence spectra $\left(\lambda_{\mathrm{ex}}=365 \mathrm{~nm}\right)$ of three luminous paints with green, blue and red emission, respectively, as well as that of the Eu-PNIPAM, Tb-PNIPAM and DAEANPNIPAM hydrogels. The Eu-PNIPAM and Tb-PNIPAM hydrogels are nearly non-fluorescent under $365 \mathrm{~nm}$ light excitation, because the picolinate chromophore of K6APA ligand has only a small conjugation unit that has no absorbance band above $300 \mathrm{~nm}$. Therefore, the device displayed the color of fluorescent paint under $365 \mathrm{~nm}$ UV light. When a voltage was applied, the recorded luminescence intensity was significantly reduced, which further demonstrated that the emission light of the luminous paint layer could be blocked by the phase-transitioned hydrogel layer. The AIE-active monomer, DAEAN, also emitted bright blue fluorescence under $365 \mathrm{~nm}$ UV light. Therefore, the color-tunable pattern of DAEAN-PNIPAM hydrogel-graphene system under $365 \mathrm{~nm}$ illumination was similar to that of $254 \mathrm{~nm}$. 


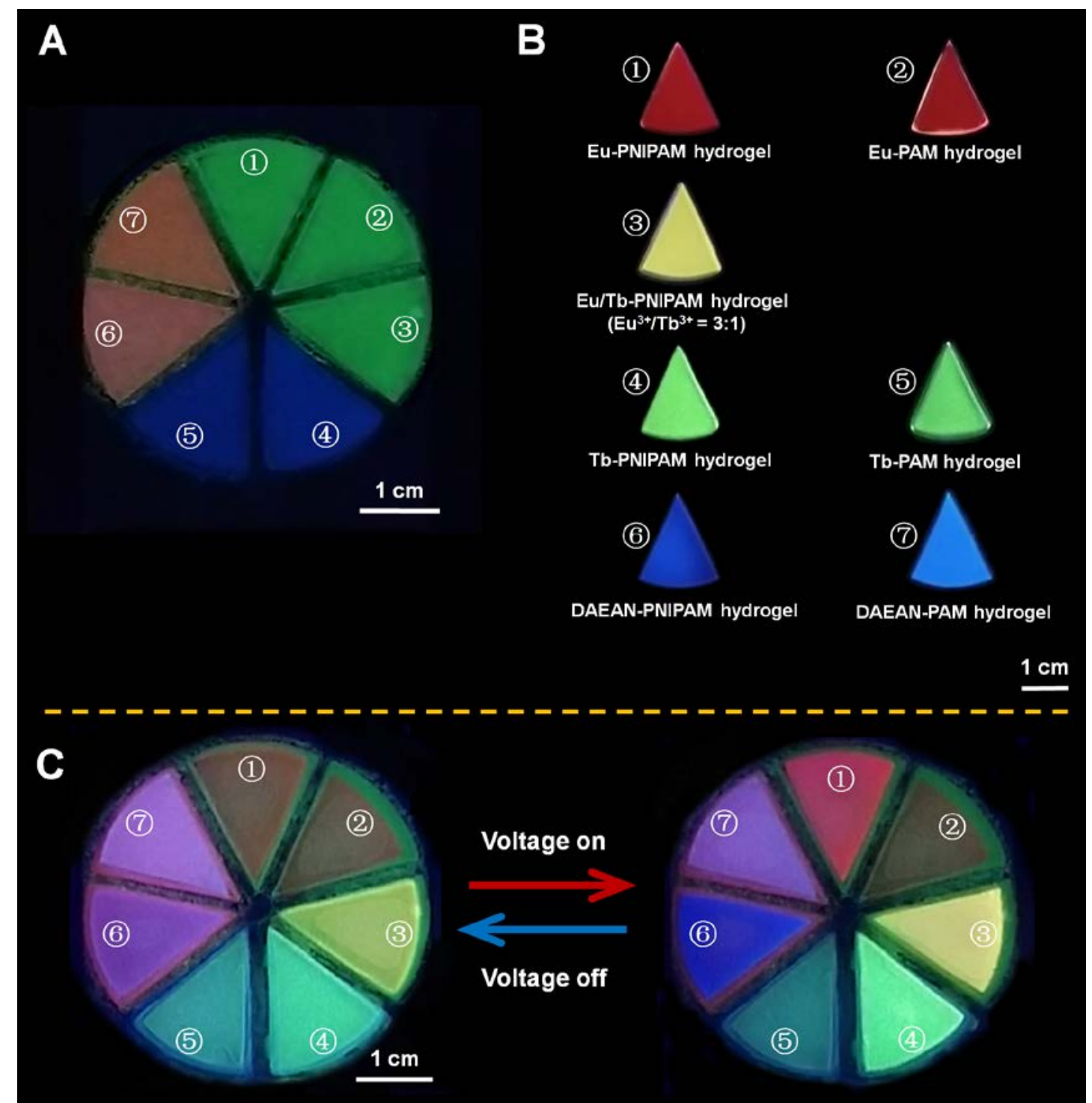

Figure S19. A colorful disk showing stunning rainbow colors under applied voltage. (A) Images of a disk with seven sectors composed of red, green and blue luminous paint. (B) Photographs of different-colored fluorescent hydrogels. (C) A colorful disk with seven sectors displaying stunning rainbow colors under the stimulus of applied voltage. Each part of the disk was prepared by combining the fluorescent hydrogel layer and luminous paint layer according to the marked numbers. For example, the Eu-PNIPAM hydrogel was covered on the position (1), while the Eu-PAM hydrogel was covered on the position (2), and so on. All photos were taken under a $254 \mathrm{~nm}$ UV lamp. 

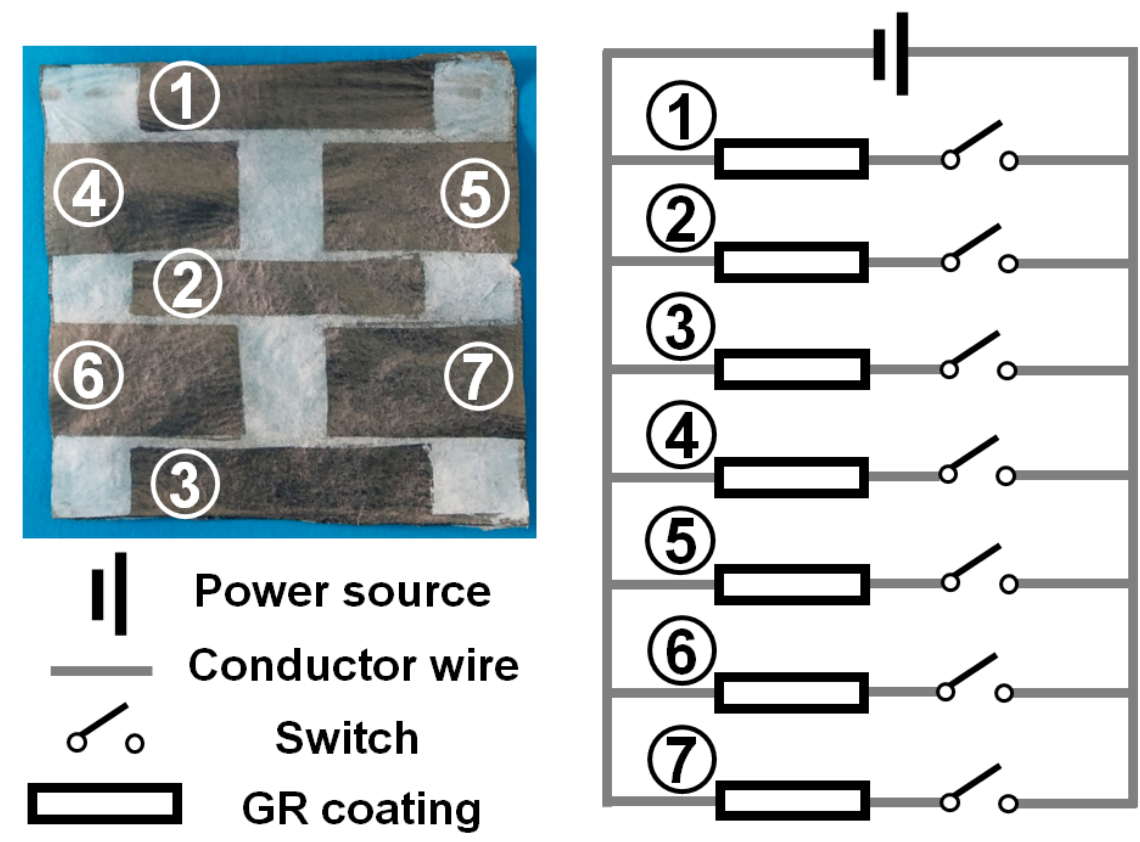

Figure S20. Photograph of the patterned SGA film and schematic illustration of the corresponding circuit.

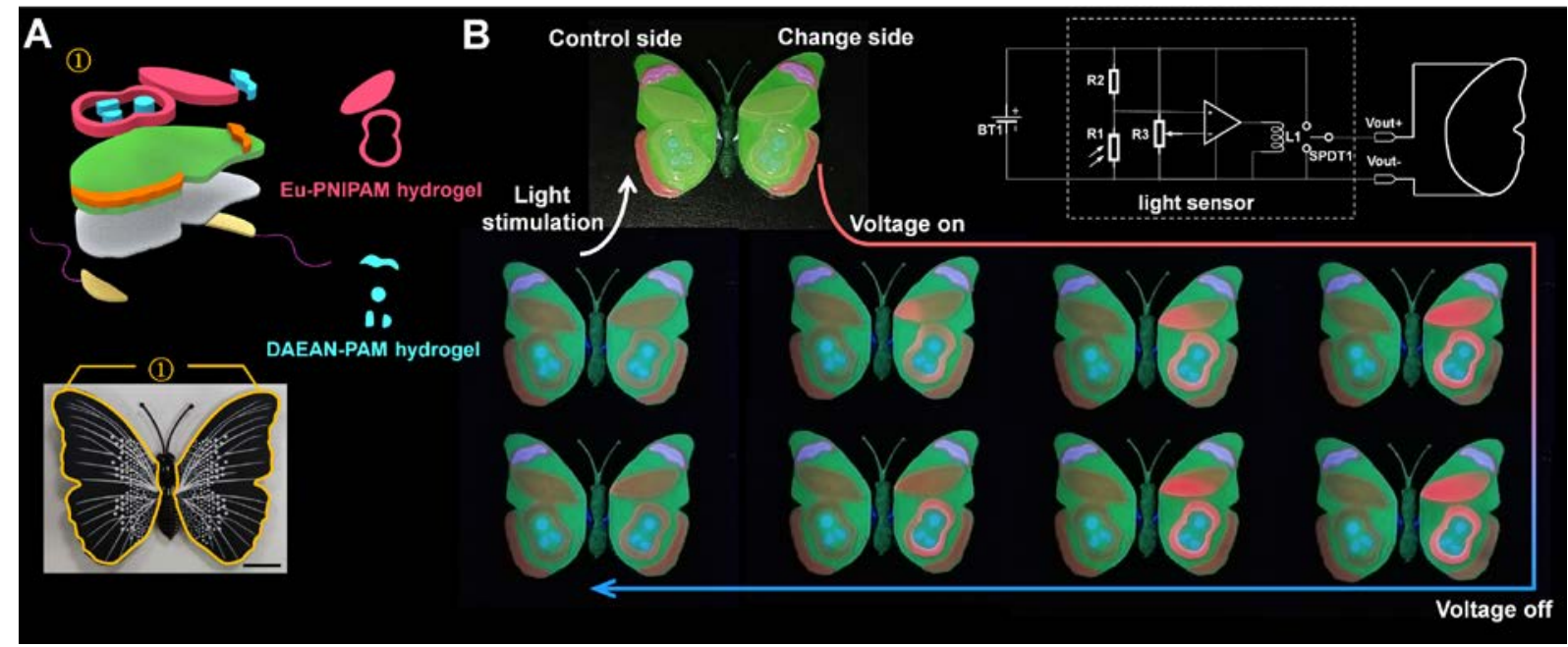

Figure S21. Application as the biomimetic skin that helps the artificial butterfly realize the alarming function. (A) Illustration showing the multilayered structure of the artificial butterfly that integrated electrically controlled fluorescent color changeable hydrogel-graphene system and butterfly model. (B) Photos showing the dynamic alarming function of the artificial butterfly. The light sensor was connected to the system to control the power on-off of the circuit. The left side of the butterfly is the control side that can facilitate the better observation of the color-changing side. Scale bar in digital photos is $1 \mathrm{~cm}$. All photos were taken under a $254 \mathrm{~nm}$ UV lamp. 